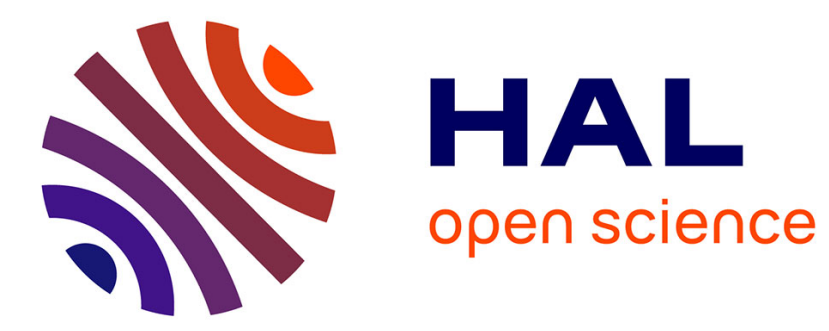

\title{
Phenomenologie et caractérisation des supraconducteurs à haute Tc
}

P. Peyral, C. Lebeau, J. Rosenblatt, Jean-Philippe Burin, A. Raboutou, O. Pena, C. Perrin

\section{- To cite this version:}

P. Peyral, C. Lebeau, J. Rosenblatt, Jean-Philippe Burin, A. Raboutou, et al.. Phenomenologie et caractérisation des supraconducteurs à haute Tc. Journal de Physique III, 1991, 1 (11), pp.1815-1821. 10.1051/jp3:1991234 . jpa-00248704

\section{HAL Id: jpa-00248704 https://hal.science/jpa-00248704}

Submitted on 1 Jan 1991

HAL is a multi-disciplinary open access archive for the deposit and dissemination of scientific research documents, whether they are published or not. The documents may come from teaching and research institutions in France or abroad, or from public or private research centers.
L'archive ouverte pluridisciplinaire HAL, est destinée au dépôt et à la diffusion de documents scientifiques de niveau recherche, publiés ou non, émanant des établissements d'enseignement et de recherche français ou étrangers, des laboratoires publics ou privés. 
Classification

Physics Abstracts

74.70B

\title{
Phénoménologie et caractérisation des supraconducteurs à haute $\boldsymbol{T}_{\mathrm{c}}$
}

\author{
P. Peyral ( ${ }^{\text {l) }}$, C. Lebeau ('), J. Rosenblatt ( $\left.{ }^{1}\right)$, J. P. Burin ('), A. Raboutou ('), \\ Q. Pena $\left({ }^{2}\right)$ et C. Perrin ( $\left.{ }^{2}\right)$ \\ (') Laboratoire de Physique des Solides URA 786 au CNRS, INSA, B.P. 14A, F-35043 Rennes \\ Cedex, France \\ (2) Laboratoire de Chimie Minérale B, URA 754 au CNRS, Avenue du Général Leclerc, F- \\ 35042 Rennes Cedex France
}

(Reçu le 25 mars 1991, accepté le 10 juillet 1991)

\begin{abstract}
Résumé. - Les propriétés de transport des supraconducteurs dépendent à la fois de la structure microscopique du matériau idéal et des caractéristiques mésoscopiques (la granularité) de chaque échantillon. Du point de vue expérimental il est essentiel de pouvoir séparer leurs effets respectifs. Nous parvenons à cette séparation en décrivant quantitativement la transition résistive sous champ nul en deux étapes: supraconductrice intragranulaire et cohérente intergranulaire. Des théories connues des fluctuations critiques et gaussiennes nous permettent alors d'obtenir des paramètres importants pour la caractérisation du matériau intragranulaire, comme sa température critique, sa résistivité normale et la largeur de sa région critique.
\end{abstract}

\begin{abstract}
Transport properties of high $T_{\mathrm{c}}$ superconductors depend on the microscopic structure of the ideal material and on the mesoscopic characteristics of each sample. From an experimental point of view it is essential to separate their effects. This can be done by describing quantitatively the resistive transition in zero field as a two-step process: a superconducting intragranular transition and an intergranular coherence transition. Well-known theories of critical fluctuations and Gaussian fluctuations allow us to obtain important characteristic parameters of the intragranular material such as the critical temperature, the normal resistivity and the width of the critical region.
\end{abstract}

\section{Introduction.}

Les plus ou moins bonnes propriétés de transport des matériaux supraconducteurs - courant critique, résistivité normale, effets du champ magnétique... - apparaissent très souvent corrélées avec la forme de la transition résistive $\rho(T)$. La mauvaise qualité se traduit par une transition "arrondie » et " élargie ". Le but de ce travail est en fait de décrire ces deux effets de façon quantitative dans le cas des supraconducteurs à haute température (SHT).

L'arrondi des transitions résistives des supraconducteurs à basse température (SBT) est dû à des petites fluctuations (gaussiennes) du paramètre d'ordre au-dessus de la température critique supraconductrice $T_{\mathrm{cs}}$. L'application des modèles existants dans leurs versions $2 \mathrm{D}$ ou 
3D [1], ou dans leur développement pour des systèmes très anisotropes [2] s'est heurtée à des difficultés dans le cas des SHT [3-7]. En général des facteurs de correction [4, 5] sont introduits pour obtenir un accord relativement satisfaisant. Nous avons pu montrer [8] que ces difficultés disparaissent quand on tient compte des effets de la granularité. L'étude de la résistivité $\rho(T)$ pour $T>T_{\text {cs }}$ nous a permis ainsi de montrer que les fluctuations correspondent à des plans $\mathrm{CuO}_{2}$ supraconducteurs faiblement couplés. Nous obtenons la longueur de cohérence $\xi_{\mathrm{c}}(0)$ perpendiculaire aux plans et leur distance $d$. Cette dernière est en très bon accord avec les mesures cristallographiques.

L'élargissement de la transition des SBT granulaires est directement lié à la formation de barrières intergranulaires et à l'augmentation de résistivité qui en résulte [9]. Dans le cas particulier où la taille des grains $a>\xi_{\mathrm{s}}(T)$, ( $\xi_{\mathrm{s}}$ est la longueur de cohérence du supraconducteur homogène) nous avons montré $[10,11]$ que la partie élargie n'est rien d'autre que la région critique, devenue observable, d'une nouvelle transition de phase, la transition à la cohérence entre grains à une température $T_{\mathrm{c}}<T_{\mathrm{cs}}$.

Dans ce qui suit nous discutons d'abord les effets de la granularité pour les supraconducteurs en général (Sect. 2). La section 3 présente des résultats sur la transition résistive des SHT en l'absence de champ et contient en particulier ce que nous pensons être la première vérification expérimentale du critère de Ginzburg [12]. Elle nous permet d'obtenir la largeur de la région critique du matériau intragranulaire. La section 4 décrit les effets de la granularité sur l'《arrondi » de la courbe. Une discussion de l'intérêt de ces résultats pour la caractérisation des SHT est proposée dans la section 5.

\section{Granularité et longueurs caractéristiques.}

Rappelons un modèle très simple des supraconducteurs granulaires [13]. Il suppose que la fonctionnelle de Ginzburg-Landau (GL) se transforme d'après

$$
\begin{gathered}
\int \mathrm{d}^{3} \mathbf{r}\left[A|\Delta|^{2}+\frac{B}{2}|\Delta|^{4}\right] \rightarrow F_{\mathrm{G}}=\sum_{i} a^{3}\left[A\left|\Delta_{i}\right|^{2}+\frac{B}{2}\left|\Delta_{i}\right|^{4}\right] \\
\int \mathrm{d}^{3} \mathrm{r} C|\nabla \Delta|^{2} \mapsto F_{\mathrm{J}}=\frac{1}{2} \sum_{\langle i\rangle} C_{i j}\left|\Delta_{i}-\Delta_{j}\right|^{2}
\end{gathered}
$$

quiand le matériau devient granulaire avec des grains de taille $a$. Une condition nécessaire pour la validité de cette transformation est que le paramètre d'ordre $\Delta_{i}=\left|\Delta_{i}\right| \exp \left(i \varphi_{i}\right)$ soit constant dans le volume du grain $i$. La condition sera satisfaite dans deux limites intéressantes :

a) petits grains (0D) [13] avec $a \ll \xi_{\mathrm{s}}(T)$, où $\xi_{\mathrm{s}}(T)=(C /|A|)^{1 / 2}$ est la longueur de cohérence du matériau intragranulaire;

b) gros grains (3D) $[11,14]$ avec $a \gg \xi_{s}(T)$ pourvu que les champs et/ou courants appliqués soient suffisamment faibles pour ne pas perturber le paramètre d'ordre dans le grain et que l'énergie de couplage intergranulaire $J_{i j} \approx C_{t j}\left|\Delta_{i}\right|\left|\Delta_{j}\right|$ soit négligeable vis-à-vis de l'énergie de condensation du grain, $F_{1} \simeq a^{3} A^{2} / 2 B$. Tenant compte de la valeur d'équilibre $\Delta_{0}^{2}=|A| / B$ la dernière condition devient :

$$
a^{2} \gg \frac{2\left\langle C_{i j}\right\rangle}{a|A|}=4 \xi_{\mathrm{MF}}^{2}=\frac{a^{2}\left\langle J_{i j}\right\rangle}{F_{1}}
$$

La nouvelle longueur de cohérence $\xi_{\mathrm{MF}}(T)$ correspond à un traitement en champ moyen de la granularité [13], une fois que l'énergie libre GL est transformée d'après (1) et (2). 
Corrélativement la température critique est aussi modifiée par le couplage Josephson (ou plutôt par le découplage par rapport au système homogène) entre grains. La nouvelle température critique $T_{\mathrm{c}}<T_{\mathrm{cs}}$ ne peut pas être calculée à partir d'une simple théorie de champ moyen. Dans le cas de gros grains et d'un faible couplage $a \gg \xi_{\mathrm{s}}(T)>\xi_{\mathrm{MF}}(T)$ nous avons montré que la température $T_{\mathrm{c}}$ marquait l'établissement de la cohérence de phase entre grains dont la transition supraconductrice a lieu à $T_{c s}$. Le paramètre d'ordre de la transition à la cohérence est défini par la moyenne thermique $M=\langle\exp (i \varphi)\rangle$, isomorphe d'un ferromagnétique $X Y$ [11] avec des interactions à courte portée correspondant aux couplages entre grains. Comme dans toutes les transitions de phase de second ordre, il existe une longueur de corrélation intergranulaire $\xi(T) \sim \varepsilon^{-\nu}$, où $\varepsilon=\left|T-T_{\mathrm{c}}\right| / T_{\mathrm{c}}$. L'exposant $\nu=1,3$ a été mesuré $[15,16]$ dans des ensembles granulaires à couplage aléatoire. Si les grains sont des supraconducteurs $\mathrm{BCS}$ sales $\xi_{\mathrm{s}}^{2}(0) \simeq \xi_{0} \ell_{\mathrm{G}}$, où $\xi_{0}=\hbar v_{\mathrm{F}} / \pi \Delta_{0}(0)$ et $\ell_{\mathrm{G}}$ est le libre parcours moyen élastique du matériau intragranulaire, de résistivité $\rho_{\mathrm{GN}}$. On peut montrer [17] que le système granulaire est un supraconducteur de type II avec

$$
\xi_{\mathrm{MF}}^{2}(0) \simeq \xi_{0} /\left[\ell_{\mathrm{G}}^{-1}+\ell_{\mathrm{b}}^{-1}\right] \simeq \xi_{\mathrm{s}}^{2}(0) \rho_{\mathrm{GN}} / \rho_{\mathrm{N}},
$$

où $\ell_{b} \simeq$ at [18] ( $t=$ coefficient de transmission des barrières) est le libre parcours moyen associé à la résistivité $\rho_{b}(T)$, mesurée quand les grains sont des conducteurs parfaits. Autrement dit, la résistivité normale est $\rho_{\mathrm{N}}(T)=\rho_{\mathrm{GN}}(T)+\rho_{b}(T)$.

Des différences essentielles sont à souligner entre ces longueurs. D'une part $\xi(T)$ décrit la portée des corrélations de phase entre grains, de ce fait $\xi(0) \simeq a$ en ordre de grandeur. D'autre part la portée des variations du paramètre d'ordre supraconducteur du matériau homogène est $\xi_{\mathrm{s}}(T)=\xi_{\mathrm{s}}(0) \varepsilon_{\mathrm{s}}^{-1 / 2}\left(\varepsilon_{\mathrm{s}}=\left|T-T_{\mathrm{cs}}\right| / T_{\mathrm{cs}}\right)$ avec $\xi_{\mathrm{s}}(0) \ll a$ dans le cas (b), et $\xi_{\mathrm{MF}}(T)$ représente les modifications de $\xi_{s}(T)$ dues à la granularité.

\section{Universalité de la transition résistive.}

A part $T_{c s}$ les autres paramètres $T_{c}, \rho_{\mathrm{b}}, \rho_{\mathrm{GN}}$ et par conséquent $\xi(0), \xi_{\mathrm{s}}(0), \xi_{\mathrm{MF}}(0)$, $a$, peuvent varier beaucoup d'un échantillon SHT à l'autre. Or, les différents comportements dépendent surtout des rapports de $a$ aux autres longueurs caractéristiques. Ceci explique l'apparente complexité des propriétés de ces matériaux.

Considérons un matériau granulaire avec $a \gg \xi_{\mathrm{s}}(T)$. Lorsque les grains sont supraconducteurs $\left(T<T_{\text {cs }}\right.$ ) les fluctuations de phase empêchent l'effet Josephson de rendre l'échantillon supraconducteur à $T>T_{\mathrm{c}}$. Dans la région critique il apparaît plutôt un excès de conductivité [19] :

$$
\sigma_{\mathrm{XY}}(T)=\sigma(T)-\sigma_{\mathrm{b}} \simeq \varepsilon^{-\gamma}
$$

où $\gamma=2,7$ a été mesuré sur des ensembles granulaires. La résistivité résiduelle $\sigma_{\mathrm{b}}^{-1}=\rho_{\mathrm{b}}\left(T_{\mathrm{cs}}\right)=\rho\left(T_{\mathrm{cs}}\right)$ est celle due aux barrières quand les grains deviennent supraconducteurs $\left(\rho_{\mathrm{G}}=0\right)$. La détermination de $\sigma_{\mathrm{b}}$ équivaut à celle de $T_{\mathrm{cs}}$. Nous l'obtenons comme le seul paramètre ajustable dans l'équation (5) quand $\left[\sigma_{\mathrm{XY}} / \sigma_{\mathrm{b}}\right]^{-1 / 2,7}$ expérimental est tracé en fonction de $T$ (Fig. 1a). L'extrapolation de la droite ainsi obtenue détermine $T_{\mathrm{c}}$. La résistivité normale $\rho_{\mathrm{N}}(T)$ peut être estimée par extrapolation linéaire de $\rho(T)$ à haute température.

L'intervalle de température où la loi (5) est satisfaite est la région critique paracohérente. Comme le montre la figure 1a elle varie d'un échantillon à l'autre. Cependant, cette variation devient compréhensible à la lumière du critère de Ginzburg [12]. Rappelons que ce critère 

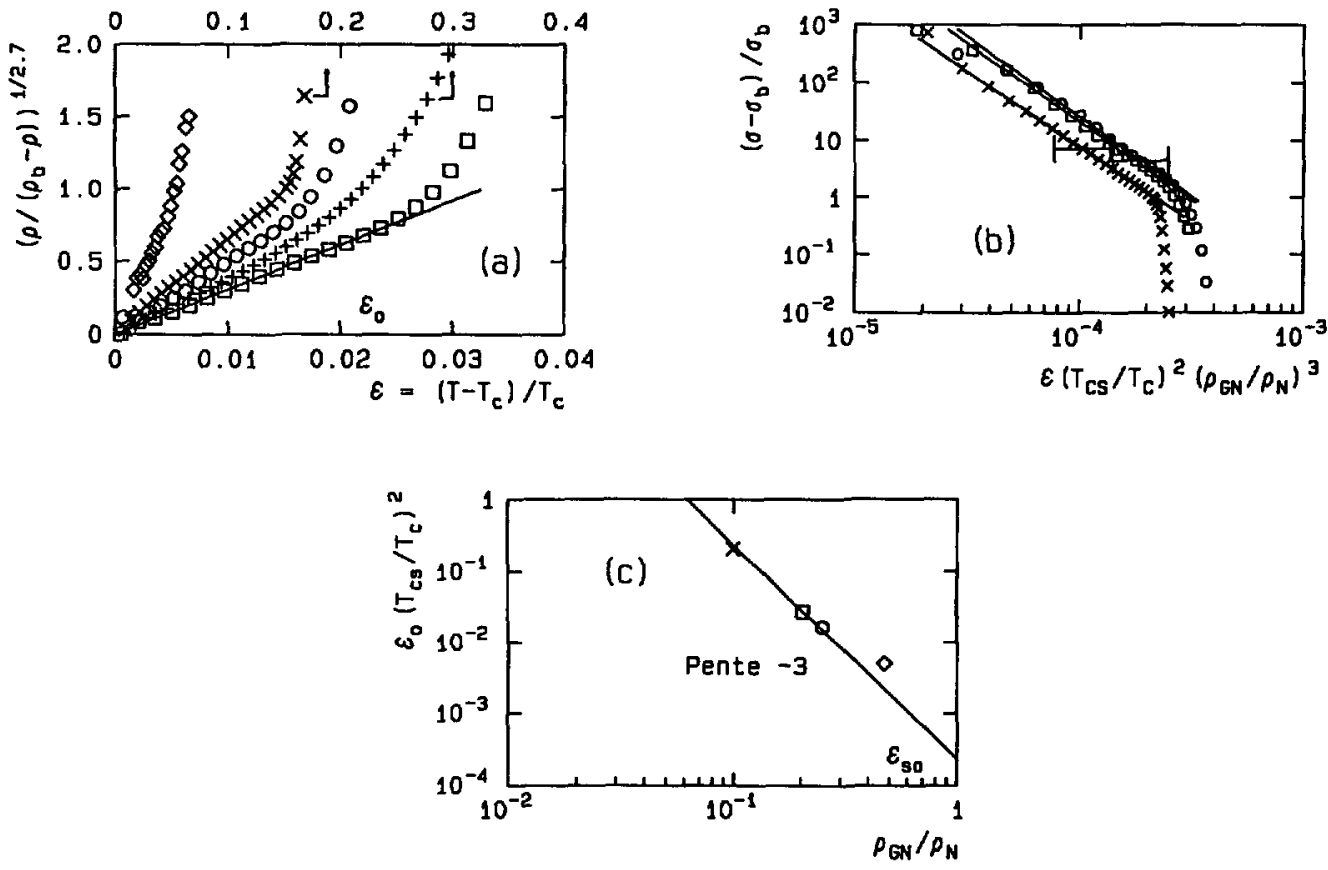

Fig. 1. - Comportement critique de la résistivité de céramiques $\mathrm{YBa}_{2} \mathrm{Cu}_{3} \mathrm{O}_{7-x}$ caractérisées par :

\begin{tabular}{clcc} 
& $\rho_{\mathrm{GN}} / \rho_{\mathrm{N}}$ & \multicolumn{1}{c}{$T_{\mathrm{cs}}$} & $T_{\mathrm{c}}$ \\
$\times$ & 0,1 & $90,9 \mathrm{~K}$ & $76,9 \mathrm{~K}$ \\
$\square$ & 0,205 & $90,6 \mathrm{~K}$ & $86,9 \mathrm{~K}$ \\
0 & 0,25 & $90,6 \mathrm{~K}$ & $89,4 \mathrm{~K}$ \\
$\diamond$ & 0,43 & $92,4 \mathrm{~K}$ & $91,7 \mathrm{~K}$
\end{tabular}

a) Détermination de la largeur critique paracohérente $\varepsilon_{0}$. Les résultats obtenus sur un SBT granulaire de $\mathrm{Ta}\left(+, \rho_{\mathrm{GN}} / \rho_{\mathrm{N}} \simeq 10^{-4}, T_{\mathrm{c}}=2,7 \mathrm{~K}\right)$ illustrent la similarité de comportement avec les SHT. b) Universalité de la transition pour un même matériau intragranulaire. c) Détermination de la région critique intragranulaire $\varepsilon_{\mathrm{s} 0}$.

[Resistivity behaviour of $\mathrm{YBa}_{2} \mathrm{Cu}_{3} \mathrm{O}_{7-8}$ ceramics. a) Paracoherent critical width $\varepsilon_{0}$. b) Universality of the transition for the same material. c) Evaluation of the intragranular critical region $\varepsilon_{\mathrm{s} 0}$.]

fournit une estimation de l'étendue de la région critique, appliqué à un supraconducteur homogène $3 \mathrm{D}$ il devient

$$
\varepsilon_{\mathrm{s} 0}^{\prime}=\left[\frac{k_{\mathrm{B}} T_{\mathrm{cs}}}{F(0) \xi_{\mathrm{s}}{ }^{3}(0)}\right]^{2}
$$

Pour $\varepsilon_{\mathrm{s}} \gg \varepsilon_{\mathrm{s} 0}^{\prime}$ la théorie de GL est valable et le comportement devient véritablement critique pour $\varepsilon_{\mathrm{s}} \ll \varepsilon_{\mathrm{s} 0}^{\prime}$. Or, dans les $\mathrm{SBT} \varepsilon_{\mathrm{s} 0}^{\prime} \sim 10^{-10}-10^{-12}$ et la région critique n'est pas observable. Une augmentation de résistivité de plusieurs ordres de grandeur serait nécessaire d'après (6) pour la rendre accessible par la diminution de $\xi_{\mathrm{s}}(0)$. Une telle augmentation de $\rho_{\mathrm{N}}$ est possible dans les SBT granulaires. En effet, il y a un élargissement de la transition [9] qui augmente avec la résistivité, mais cette observation reste qualitative. 
La petitesse de $\xi_{\mathrm{s}}(0)$ dans les SHT suggère que de faibles variations de $\rho_{\mathrm{N}}$ auront des effets bien plus spectaculaires. Leur description la plus simpliste consiste à remplacer $T_{c s}$ par $T_{\mathrm{c}}$ et $\xi_{\mathrm{s}}(0)$ par $\xi_{\mathrm{MF}}(0)$ dans l'équation (6), ce qui donne :

$$
\varepsilon_{0}^{\prime}=\left[\frac{k_{\mathrm{B}} T_{\mathrm{c}}}{F(0) \xi_{\mathrm{MF}}^{3}(0)}\right]^{2}
$$

Finalement, si l'on suppose que les largeurs critiques effectivement mesurées (voir Fig. 1a), $\varepsilon_{\mathrm{s} 0}$ et $\varepsilon_{0}$, sont proportionnelles à $\varepsilon_{\mathrm{s} 0}^{\prime}$ et $\varepsilon_{0}^{\prime}$, respectivement, on obtient, compte tenu de (4)

$$
\varepsilon_{0}=\varepsilon_{\mathrm{s} 0}\left[\frac{T_{\mathrm{c}}}{T_{\mathrm{cs}}}\right]^{2}\left[\frac{\rho_{\mathrm{N}}}{\rho_{\mathrm{GN}}}\right]^{3}
$$

Les données de la figure la forment une seule courbe universelle quand on remplace $\varepsilon \operatorname{par} \varepsilon / \varepsilon_{0}$. De la même manière, plusieurs échantillons donneront une courbe universelle pour $\sigma_{\mathrm{XY}} / \sigma_{\mathrm{b}}$ en fonction de $\varepsilon \quad \varepsilon_{\mathrm{s} 0} / \varepsilon_{0}$ si leur matériau intragranulaire a le même $\varepsilon_{\mathrm{s} 0}$. Cette prédiction est confirmée par la figure $1 \mathrm{~b}$, où on a utilisé l'expression (8) pour $\varepsilon_{\mathrm{s} 0} / \varepsilon_{0}$. La figure $1 \mathrm{c}$ est une vérification directe de l'équation (8), illustrant l'obtention $\mathrm{du}$ paramètre $\varepsilon_{\mathrm{s} 0}$ pour une série d'échantillons.

Ces résultats constituent la première preuve expérimentale du critère de Ginzburg.

\section{Paraconductivité.}

Les fluctuations de l'amplitude du paramètre d'ordre à $T>T_{\mathrm{cs}}$ donnent lieu au phénomène de la paraconductivité [1]. Dans un modèle des supraconducteurs anisotropes [2] l'excès de conductivité est donné par:

$$
\sigma_{\mathrm{s}}=g /\left[\mathrm{d}^{2} \varepsilon_{\mathrm{s}}^{2}+4 \xi_{\mathrm{c}}^{2}(0) \varepsilon_{\mathrm{s}}\right]^{1 / 2}
$$

où $g=e^{2} / 16 \hbar=1,52 \times 10^{-5} \Omega^{-1}, d$ est la distance entre plans et $\xi_{\mathrm{c}}(0)$ la longueur de cohérence à $T=0$ dans la direction perpendiculaire aux plans supraconducteurs. La comparaison de la différence de conductivité $\delta \sigma=\sigma(T)-\sigma_{\mathrm{N}}(T)$ expérimentale avec $\sigma_{\mathrm{s}}$ donnée par l'équation (9) s'est avérée décevante [4-6], et cela malgré le fait que $d, \xi_{\mathrm{c}},(0), T_{\mathrm{cs}}$ et les paramètres définissant $\sigma_{\mathrm{N}}(T)$ soient ajustables. En général on obtient $\sigma_{\mathrm{s}}=C \delta \sigma$, où $C \approx 5-7$ est un facteur de correction qui varie avec l'échantillon. Une telle correction est tout à fait contraire au modèle lui-même.

Nous avons montré que ces difficultés disparaissent quand on tient compte de la granularité $[8,20]$. En effet, la résistivité est :

$$
\rho(T)=\rho_{\mathrm{G}}(T)+\rho_{\mathrm{b}}(T),
$$

tandis que la résistivité due aux barrières $\rho_{\mathrm{b}}$ n'est pas affectée par les fluctuations supraconductrices à $T>T_{\mathrm{cs}}$. La paraconductivité est donc localisée dans les grains dont la conductivité normale est $\sigma_{\mathrm{GN}}=1 / \rho_{\mathrm{GN}}$. A partir de l'équation (10) et de l'expression de la conductivité des grains :

$$
\sigma_{\mathrm{G}}=1 / \rho_{\mathrm{G}}=\sigma_{\mathrm{GN}}+\sigma_{\mathrm{s}},
$$


on obtient la différence de résistivités mesurée expérimentalement :

$$
\delta \rho=\rho_{\mathrm{N}}(T)-\rho(T)=\frac{\sigma_{\mathrm{s}} \rho_{\mathrm{GN}}^{2}}{1+\sigma_{\mathrm{s}} \rho_{\mathrm{GN}}}
$$

Dans la région de températures où $\sigma_{\mathrm{s}} \rho_{\mathrm{GN}} \ll 1, \delta \sigma=\delta \rho / \rho \rho_{\mathrm{N}} \approx \sigma_{\mathrm{s}}\left(\rho_{\mathrm{GN}} / \rho_{\mathrm{N}}\right)^{2}$, ce qui permet d'identifier le facteur de correction $C \simeq\left(\rho_{\mathrm{N}} / \rho_{\mathrm{GN}}\right)^{2}$. La figure 2 montre nos résultats pour deux échantillons de $\mathrm{YBa}_{2} \mathrm{Cu}_{3} \mathrm{O}_{7-x}$. En représentant $g^{2} / \varepsilon_{\mathrm{s}} \sigma_{\mathrm{s}}^{2}$ en fonction de $\varepsilon_{\mathrm{s}}$ on doit obtenir, d'après l'équation (9), une droite de pente $d^{2}$ et d'ordonnée à l'origine $4 \xi_{\mathrm{c}}^{2}(0)$. Les points expérimentaux indiqués dans la figure 2 proviennent des valeurs mesurées de $\delta \rho$ remplacées dans l'équation (12). Il est à signaler qu'à part la droite elle-même, aucun paramètre n'est ajustable dans la figure 2 . En effet, les valeurs de $T_{\mathrm{cs}}, \rho_{\mathrm{b}}\left(T_{\mathrm{cs}}\right)=\rho\left(T_{\mathrm{cs}}\right)$, et $\rho_{\mathrm{GN}}=\rho_{\mathrm{N}}-\rho_{\mathrm{b}}$ sont obtenues par l'étude de la région critique $T_{\mathrm{c}} \leqslant T<T_{\mathrm{cs}}$ (Sect. 3). Pour $T>T_{\mathrm{cs}}$ nous supposons $\rho_{\mathrm{GN}} / \rho_{\mathrm{N}}=$ constante.

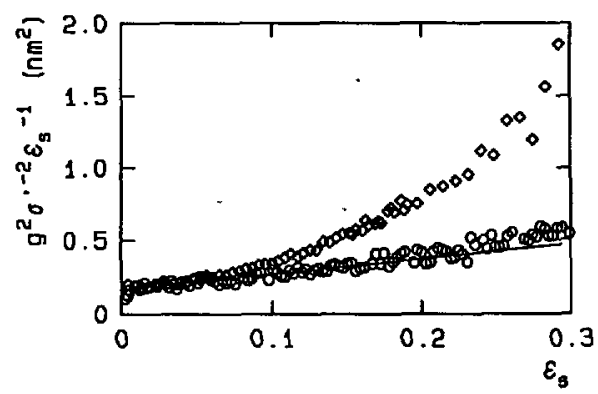

Fig. 2. - Paraconductivité d'échantilllons frittés $\mathrm{YBa}_{2} \mathrm{Cu}_{3} \mathrm{O}_{7-x} \cdot(\diamond) \rho_{\mathrm{GN}} / \rho_{\mathrm{N}}=0,43, T_{\mathrm{cs}}=92,4 \mathrm{~K} ;(\mathrm{O})$ $\rho_{\mathrm{GN}} / \rho_{\mathrm{N}}=0,25, T_{\mathrm{cs}}=90,6 \mathrm{~K}$. La droite correspond à l'équation (9) avec $d=1,2 \mathrm{~nm}$ et $\xi_{\mathrm{c}}(0)=0,2 \mathrm{~nm}$. [Paraconductivity of sintered $\mathrm{YBa}_{2} \mathrm{Cu}_{3} \mathrm{O}_{7-\delta}$ samples.]

\section{Discussion.}

Nous analysons la transition résistive comme un processus en deux étapes: transition intragranulaire à $T_{\mathrm{cs}}$, intergranulaire à $T_{\mathrm{c}}$. Très qualitativement, ces étapes correspondent à l'« onset » et l'« offset » de certains auteurs. L'étude quantitative de ce processus conduit à la détermination de plusieurs paramètres importants pour caractériser la qualité d'un échantillon : $\rho_{\mathrm{GN}}, \rho_{\mathrm{b}}, T_{\mathrm{cs}}$ et plus particulièrement $\varepsilon_{\mathrm{s} 0}$. Ce dernier, largeur critique relative du matériau intragranulaire, semble être typique du processus de fabrication et donc commun à plusieurs échantillons, comme le montre la figure 1c. Nous le proposons en conséquence comme un élément de diagnostic de la qualité de l'élaboration.

\section{Bibliographie}

[1] Azlamazov L. G. and Larkin A. I., Fiz. Tverd. Tela 10 (1968) 1104 (Engl. Trans. : Sov. Phys. Solid State 10 (1968) 875, Phys. Lett. 26A (1968) 238.

[2] Lawrence W. E. and Doniach S., Proc. of the 12th International Conference on Low Temperature Physics, Kyoto 1970, E. Kanda Ed. (Keigaku, Tokyo, 1970) p. 361.

[3] Freitas P. P., Tsuei C. C. and Plaskett T. S., Phys. Rev. B 36 (1987) 833.

[4] Matsuda Y., Hirai T. and KomiYama S., Solid State Commun. 68 (1988) 103. 
[5] Oh B., Char K., Kent A. D., Naito M., Beasley H. R., Geballe T. H., Hammond R. H., Kapiltunik A. and Graybeal J. M., Phys. Rev. B 37 (1988) 7861.

[6] Friedmann T. A., Rice J. P., Giapintzakis J. and Ginsberg D. M., Phys. Rev. B 39 (1989) 4258.

[7] Kim D. H., Goldman A. M., Kang J. H., Gray K. E. and Kampwirth R. T., Phys. Rev. B 39 (1989) 12275.

[8] Rosenblatt J., Raboutou A., Peyral P. and Lebeau C., Revue Phys. Appl. 25 (1990) 73.

[9] Deutscher G., Fenichel H., Gershenson .M., Grumbaum E. and Ovadyahu Z., J. Low Temp. Phys. 10 (1973) 231.

[10] Pellan P., Dousselin G., Cortes H. and Rosenblatt J., Solid State Commun. 11 (1972) 427.

[11] Rosenblatt J., Raboutou A. and Pellan P., Low Temperature Physics, LT14, M. Krusius and M. Vuorio Eds., vol. 2 (American Elsevier, New York, 1975) p. 361.

[12] Ginzburg V. L., Fiz. Tverd. Tela 2 (1960) 2031 (Sov. Phys. Solid St. 2 (1960) 1824.

[13] Deutscher G. and Imry Y., Phys. Lett. 42A (1973) 413 ; DeutScher G., IMrY Y. and Gunther L., Phys. Rev. B 10 (1974) 4598.

[14] Rosenblatt J., Percolation, Localization and Superconductivity, NATO ASI series, A. M. Goldman and S. A. Wolf Eds. (Plenum, New York, 1984) p. 431.

[15] Raboutou A., Rosenblatt J. and Peyral P., Phys. Rev. Lett. 45 (1980) 1035.

[16] Rosenblatt J., Lebeau C., Peyral P. and Raboutou A., Josephson Effect - Achievements and Trends, A. Barone Ed. (World Scientific, Singapore, 1986) p. 320.

[17] Rosenblatt J., Burin J. P., Raboutou A., Peyral P. and Lebeau C., Phase Transitions 30 (1991) 157.

[18] Parmenter R. H., Phys. Rev. 154 (1967) 353.

[19] Rosenblatt J., Peyral P. and Raboutou A., Phys. Lett. 98A (1983) 463;

Lebeau C., Rosenblatt J., Raboutou A. and Peyral P., Europhys. Lett. 1 (1986) 313.

[20] Raboutou A., Peyral P., Lebeau C., Rosenblatt J. and Burin J. P., J. Less. Common Metals 164 \& 165 (1990) 1400. 


\section{Articles Réguliers}

